\title{
Les effets in vivo de certains produits géniques ne sont pas comparables à ceux qu'on leur connaît in vitro
}

\begin{abstract}
Depuis de nombreuses années se sont accumulées les connaissances relatives aux effets de nombreuses protéines sur la différenciation et la régulation du cycle cellulaire. Le clonage de leurs gènes et leur expression ont permis de disposer de protéines recombinantes purifiées et d'en étudier les fonctions. Très rapidement, de nombreux laboratoires se sont attachés à vérifier si les effets connus in vitro des facteurs de croissance, en particulier hématopoïétiques, pouvaient être retrouvés chez l'animal après injection du facteur ou expression déréglée du gène dans des souris transgéniques. Dans les divers cas rapportés, une bonne corrélation a été observée : l'injection d'érythropoiétine stimule l'activité érythropoiétique, celle du GM-CSF (granulocyte-macrophagecolony stimulating factor) la production de cellules myéloïdes, pour ne citer que ces exemples. Cependant, les expériences de Metcalf et de ses collaborateurs à Melbourne (Autralie) avaient démontré que l'excès de GM-CSF dans des souris transgéniques pouvait avoir des conséquences dramatiques pour l'animal [1].
\end{abstract}

Pour être complète, l'analyse du rôle d'un gène doit aussi se réaliser chez des animaux privés du gène fonctionnel. Pour cela, des cellules souches embryonnaires (ES) de souris sont soumises à une recombinaison homologue visant à intégrer un fragment altéré d'un gène en lieu et place de son homologue normal [2]. Les cellules ES sélectionnées sont alors utilisées pour obtenir des souris chimères puis des souris hétérozygotes et enfin homozygotes pour le gène muté. L'obtention des souris démunies d'un gène fonctionnel permet alors a contrario de préciser l'importance relative du gène dans l'organisme.

Des études récentes viennent d'appor- gènes codant respectivement pour le LIF (leukemia inhibitory factor), la protéine p53 et la protéine $\mathrm{Rb}$ du gène de susceptibilité au rétinoblastome.

Le LIF est une cytokine dont les effets in vitro sont très variés et viennent d'être rappelés en détail dans $m / s$ [3] : il inhibe la différenciation des cellules ES, des cellules rénales, des préadipocytes mais induit la différenciation des cellules leucémiques M1, favorise la survie et la prolifération des cellules D1a, des progéniteurs hématopoiétiques, des cellules germinales primordiales et de certains neurones, inhibe la résorption osseuse par les ostéoclastes et induit la production des protéines de la phase aiguë par des cellules du parenchyme hépatique [3-5]. L'injection de fortes doses du LIF à des souris se traduit par une perte de poids due à la disparition des dépôts graisseux abdominaux et sous-cutanés, une atrophie du thymus, une augmentation du calcium sérique, du nombre des progéniteurs mégacaryocytiques et du compte plaquettaire [6]. Des souris LIF-/-complètement dépourvues de cette cytokine viennent d'être obtenues et sont parfaitement viables [7] malgré les évidences d'un retard de la croissance post-natale. Ces souris sont fertiles. Le seul défaut apparent lié à l'absence de LIF est l'incapacité des femelles à assurer la nidation de l'embryon dans la paroi utérine. Cela ne peut cependant être une surprise totale au vue de la richesse en LIF de ce tissu au moment de l'implantation du blastocyste chez la souris normale ([8], $m / s n^{\circ} 8$, vol. 8, p. 873). Mais ces résultats précisent de façon très claire le rôle du LIF dans la nidation de l'embryon et ouvrent des perspectives attrayantes d'une utilisation du LIF pour faciliter l'implantation des œufs fécondés in vitro. En revanche, les animaux LIF-/- n'ont apparemment pas démontré d'anomalies " criantes " dans la formation des tissus sur lesquels le LIF est supposé agir, bien que des études plus précises soient nécessaires. Les gènes $\mathrm{p} 53$ et $\mathrm{Rb}$ codent pour des protéines nucléaires dont le rôle est de contrôler de façon négative l'activité cyclique cellulaire, ce qui leur a valu la dénomination de gènes suppresseurs de tumeurs ou encore anti-oncogènes. La tumorisation peut survenir quand ces gènes sont mutés $[9,10]$. La mutation des deux gènes $\mathrm{Rb}$ favorise l'apparition du rétinoblastome chez l'enfant [11-13]. L'introduction d'un gène sauvage dans des cellules tumorales rétablit alors certains aspects du contrôle de la division [14, 15]. Ces protéines ont en commun la capacité de fixer les protéines oncogènes E1A de l'adénovirus, T de SV40 et E7 du papillomavirus, ce qui laisse supposer que les protéines $\mathrm{p} 53$ et $\mathrm{Rb}$ s'associent dans les cellules normales avec certaines protéines de régulation, comme le facteur E2F en particulier $\left(\mathrm{m} / \mathrm{s} n^{\circ} 8\right.$, vol. 7, p. 864). Par ailleurs, p53 et $\mathrm{Rb}$ peuvent se fixer à l'ADN et ainsi réprimer ou activer certains gènes impliqués dans la régulation du cycle cellulaire (revue dans [16-18]). Le rôle de ces deux gènes paraît donc suffisamment important pour que l'on imagine que leur mutation homozygote se traduise par des effets dramatiques. Les résultats de ces expériences viennent d'être publiés.

Curieusement, la mutation homozygote du gène p53 n'aboutit à aucune altération du développement, mais $74 \%$ des souris développent à 6 mois des lymphomes et plus rarement des sarcomes ([19], $\mathrm{m} / \mathrm{s} n^{\circ} 5$, vol. 8, p. 492). En revanche, la perte fonctionnelle de $\mathrm{Rb}$ se traduit par un développement abortif [20-22]. Au cours du développement, le foetus est globalement normal jusqu'au $10^{e}$ jour environ; les 
anomalies surviennent alors, si bien qu'aucun fotus ne survit au $16^{\mathrm{e}}$ jour. Ces anomalies affectent particulièrement le tissu nerveux central et le tissu érythropoïétique. Si la production des érythrocytes nucléés primitifs par le sac vitellin apparaît normale, celle des érythrocytes anucléés d'origine hépatique apparaît fortement déficiente, si bien que, chez le fotus de 15 jours, les érythrocytes nucléés prédominent largement, contrairement à ce qui est observé chez les foetus normaux. Enfin, surprenante observation, les souris hétérozygotes $\mathrm{Rb}^{-} / \mathrm{Rb}^{+}$développent fréquemment des tumeurs hypophysaires et aucun rétinoblastome [21] contrairement à ce qui est observé chez l'homme.

Les observations rapportées pour les trois mutations homozygotes in vivo recouvrent de façon non absolue les propriétés qu'on attribue à ces gènes. Le cas du gène $\mathrm{Rb}$ est certainement celui qui se rapproche le plus de ce que l'on pouvait attendre ; cependant, la létalité survient relativement tardivement durant la vie fotale et n'affecte massivement que quelques tissus particuliers. Curieusement, la mutation p53 n'affecte pas le développement embryonnaire et celle du gène du LIF n'a pas les larges effets escomptés. Bien sûr, les mutations de la p53 et $\mathrm{Rb}$ prédisposent à la tumorisation, ce qui était attendu.

Pourquoi des effets réduits in vivo par rapport aux propriétés définies in vitro? L'explication tient vraisemblablement à la présence d'une surabondance de signaux qui peuvent suppléer à la défaillance de l'un d'eux. Cependant, les résultats suggèrent qu'il existe des tissus dans lesquels le signal défaillant apparaît jouer un rôle majeur. Il en est peut-être ainsi du LIF dans la paroi utérine, et son défaut ne permet plus l'implantation de l'embryon, de $\mathrm{Rb}$ dans la rétine humaine ou l'hypophyse de souris, de la p53 dans la lignée lymphoïde. Il est donc possible que, lors de la différenciation des tissus, le rôle des diverses protéines se définisse de façon précise. Leur mutation (ou leur absence) fait apparaître alors au grand jour leur site d'action principal et, pour ce qui est des protéines p53 et $\mathrm{Rb}$, prédispose alors, chez les souris hétérogygotes pour le déficit, les cellules de ces tissus à la dérégulation du $\mathrm{m} / \mathrm{s} n^{\circ} 10$ vol. 8, décembre 9 ? cycle cellulaire si survient un deuxième événement mutationnel, comme envisagé par Knudson [23].

J.P.B.

1. Lang RA, Metcalf D, Curthberson RA, et al. Transgenic mice expressing a hemopoietic growth factor (GM-CSF) develop accumulation of macrophages, blindness and a fatal syndrome of tissue damage, Cell 1987; 51 : 675-86.

2. Babinet C. Les cellules souches embryonnaires de souris : une voie privilégiée de transformation génétique à l'échelle de l'animal. médecine/sciences $1992 ; 8: 268-75$.

3. Jacques Y, Anegon I, Hallet MM, Godard A, Moisan JP, Soulillou JP. Les multiples facettes de la cytokine HILDA/LIF. médecine/sciences $1992 ; 8$ : 939-49.

4. Metcalf $D$. The leukemia inhibitory factor (LIF). Int J Cell Cloning 1991 ; 9 : 95-108. 5. Smith AG, Nichols J, Robertson M, Rathjen PD. Differentiating inhibiting activity (DIA/LIF) and mouse development. Dev Biol 1992 ; $151: 339-51$.

6. Metcalf D, Nicola NA, Gearing DP. Effects of injected leukemia inhibitory factor on hematopoietic and other tissues in mice. Blood 1990; 7 : 50-6.

7. Stewart CL, Kaspar P, Brunet LJ., et al. Blastocyst implantation depends on maternal expression of leukaemia inhibitory factor. Nature $1992 ; 359$ : 76-9.

8. Bhatt $\mathrm{H}$, Brunet LJ, Stewart CL. Uterine expression of leukemia inhibitory factor coincides with the onset of blastocyst implantation. Proc Natl Acad Sci USA 1991; 88 : 11408-12. 9. Horowitz JM, Park SH, Bogenmann E, et al. Frequent inactivation of the retinoblastoma anti-oncogene is restricted to a subset of human tumor cells. Proc Natl Acad Sa USA $1990 ; 87: 2775-9$

10. Cheng J, Haas M. Frequent mutations in the p53 tumor supressor gene in human leukemia T-cell lines. Mol Cell Biol $1990 ; 10$ : 5502-9.

11. Friend SH, Bernards R, Rogelj S, et al. A human DNA segment with properties of the gene that predisposes to retinoblastoma and osteosarcoma. Nature $1986 ; 323: 643-6$ 12. Lee WH, Bookstein R, Hong F, Young LJ, Shew JY, Lee EYHP, Human retinoblastoma susceptibility gene : cloning, identification and sequence. Science 1987 ; 236 : 1394-9. 13. Fung YK, Murphree AL, T'Ang A, Qian J, Hinrichs SH, Benedict WF. Structural evidence for the authenticity of the human retinoblastoma gene. Science 1986 ; $235: 1657-61$.

14. Finlay CA, Hinds PW, Levine AJ. The p53 proto-oncogene can act as a suppressor of transformation. Cell 1989; 57 : 1083-93. 15. Huang HJS, Yee JK, Shew JY, et al. Suppression of the negative phenotype by replacement of ther $\mathrm{Rb}$ gene in human cancer lines. Science $1988 ; 242$ : 1563-66. 16. Vogelstein B, Kinzler KW. p53 function and dysfunction. Cell 1992; 70: 523-6.

17. Cobrinik D, Dowdy SF, Hinds PW, Mittnacht S, Weiberg RA. The retinoblastoma protcin and the regulation of cell cycling. Trends Biochem Sci 1992; 17: 312-5.
18. Ullrich SJ, Anderson CW, Mercer WE, Appella E. The p53 tumor suppressor protein, a modulator of cell proliferation. J Biol Chem 1992 ; 267 : 15259-62.

19. Donehower LA, Harvey M. Slagle BL, et al. Mice deficient for p53 are developmentally normal but susceptible to spontaneous tumors. Nature 1992; 356 : 215-21

20. Lee EYHP, Chang CY, Hu NP, et al. Mice deficient for $\mathrm{Rb}$ are nonviable and show defects in neurogenesis and hematopoiesis. Nature 1992 ; 359 : 288-94

21. Jacks T, Fazeli A, Schmitt EM, Bronson RT, Goodell MA, Weinberger RA. Effects of an $\mathrm{Rb}$ mutation in the mouse. Nature 1992 ; 359 : 295-300.

22. Clarke AR, Mandaag ER, Van Room $\mathrm{M}$, et al. Requirement for a functional Rb-1 gene in murine development. Nature 1992 ; 359 : 328-30.

23. Knudson AG. Mutation and cancer : statistical study of retinoblastoma. Proc Natl Acad Sci USA 1971; 68 : 820-3. 